Proceedings of "The $6^{\text {th }}$ SEAMS-UGM Conference 2011"

Applied Mathematics, pp. 317-322.

\title{
A LEPSKIJ-TYPE STOPPING-RULE FOR SIMPLIFIED ITERATIVELY REGULARIZED GAUSS-NEWTON METHOD
}

\author{
AGAH D. GARNADI
}

\begin{abstract}
Iterative regularization methods for nonlinear ill-posed equations of the form $F(a)=y$, where $F: D(F) \subset X \rightarrow Y$ is an operator between Hilbert spaces $X$ and $Y$, usually involve calculation of the Fréchet derivatives of $F$ at each iterate and at the unknown solution $a^{\sharp}$. A modified form of the generalized Gauss-Newton method which requires the Fréchet derivative of $F$ only at an initial approximation $a_{0}$ of the solution $a^{\sharp}$ as studied by Mahale and Nair [11]. This work studied an a posteriori stopping rule of Lepskij-type of the method. A numerical experiment from inverse source potential problem is demonstrated.

Keywords and Phrases: Nonlinear ill-posed problem, a posteriori stopping rule, regularized
\end{abstract} Gauss-Newton.

\section{INTRODUCTION}

Nonlinear ill-posed problem usually posed as a non-linear operator equation

$$
F: D(F) \subset \mathcal{X} \rightarrow \mathcal{Y}
$$

between Hilbert spaces $\mathcal{X}$ and $\mathcal{Y}$. We assume that $F$ is one-to-one and Fréchet differentiable on its domain $D(F)$ and denote the derivative at a point $a \in D(F)$ by $F^{\prime}[a]$. Since it is ill-posed, $F$ does not have a bounded inverse.

In this work we will focus on the simplified iteratively regularized GaussNewton method (sIRGNM) which is a variant of iteratively regularized GaussNewton method (IRGNM), one of the most attractive iterative regularization methods. For an overview on iterative regularization methods for non-linear ill-posed problem, we refer to the monograph by Kaltenbacher, Neubauer, and Scherzer [10] or Bakushinsky, Kokurin, Kokurin, and Smirnova [1]. At $(n+1)-$ st iteration of 
the IRGNM, the iterate $a_{(n+1)}^{\delta} \in \mathcal{X}$ is defined as the unique global minimizer of the quadratic functional $a \mapsto\left\|F^{\prime}\left[a_{n}^{\delta}\right]\left(a-a_{n}^{\delta}\right)+F\left(a_{n}^{\delta}\right)-y^{\delta}\right\|_{\mathcal{Y}}^{2}+\alpha_{n}\left\|\left(a-a_{0}\right)\right\|_{\mathcal{X}}^{2}, n \in \mathrm{N}_{0}$. Where $a_{0} \in D(F)$ is some initial guess, and $\alpha_{n}$ is a regularization parameter, here we chose $\alpha_{n}=\alpha_{0} q^{n}$, for some $0<q<1$. The $(n+1)$-st iterate $a_{(n+1)}^{\delta}$ can be expressed in a closed form

$$
a_{(n+1)}^{\delta}:=a_{n}+\left(F^{\prime}\left[a_{n}^{\delta}\right]^{*} F^{\prime}\left[a_{n}^{\delta}\right]+\alpha_{n} I\right)^{-1} F^{\prime}\left[a_{n}^{\delta}\right]^{*}\left(y^{\delta}-F\left(a_{n}^{\delta}\right)+F^{\prime}\left[a_{n}^{\delta}\right]\left(a_{n}^{\delta}-a_{0}\right)\right) .
$$

A variant of IRGNM, where we approximate $F^{\prime}\left[a_{n}^{\delta}\right]$ by an equivalent linear operator $A$, typically by $F^{\prime}\left[a_{0}\right]$. Hence the previous formula at the $(n+1)$-st iteration, we use

$$
a_{(n+1)}^{\delta}:=a_{n}^{\delta}+\left(F^{\prime}\left[a_{0}\right]^{*} F^{\prime}\left[a_{0}\right]+\alpha_{n} I\right)^{-1} F^{\prime}\left[a_{0}\right]^{*}\left(y^{\delta}-F\left(a_{n}^{\delta}\right)+F^{\prime}\left[a_{0}\right]\left(a_{n}^{\delta}-a_{0}\right)\right) .
$$

This variant called the simplified IRGNM, which is widely used in practice, but lacking in theoretical grounds. Kaltenbacher [9] initiated studying the methods, and closely studied in details recently by Mahale \& Nair [11], Jin [8] and George [5].

One of important thing during iteration is when to terminate the steps as the error $\left\|a_{n}^{\delta}-a^{\sharp}\right\|$ experiencing deterioration as $n \rightarrow \infty$ in the presence of noise. One of the rule that widely use is the discrepancy principle, which is the iteration terminated at the index $N\left(\delta, y^{\delta}\right)$ for the first time the criteria $\left\|F\left(a_{N}\right)-y^{\delta}\right\| \leq \tau \delta$ satisfied with some parameter $\tau>1$. In $[2,3]$ the authors studied a Lepskij-type stopping rule for IRGNM with deterministic and random noise, their studies showed that both theoretically and numerically, compared to the discrepancy principle, the proposed stopping rule yields at least as good, and at some point even better results.

In this work, we examine the stopping rule to the sIRGNM, fills a gap left behind by [2] works on IRGNM and completing the works of Bauer \& Lukas [4] on extensive survey on stopping criteria in linear inverse problem.

\section{LEPSKIJ STOPPING RULE FOR DETERMINISTIC NOISE.}

To analyze the convergence of the sIRGNM, we follows analysis for IRGNM closely. After the $(n+1)$ iterations, the total error $e_{n+1}=a_{n+1}^{\delta}-a^{\sharp}$ is decomposed into three components which are estimated one-by-one: the (modified) approximation error $e_{n+1}^{a p p}:=\alpha_{n}\left(F^{\prime}\left[a_{0}\right]^{*} F^{\prime}\left[a_{0}\right]+\alpha_{n} I\right)^{-1} e_{0}$, the (modified) propagated data noise error $e_{n+1}^{n o i}$, and the (modified) nonlinearity error $e_{n+1}^{n o i}$.

The non-linearity component error always bounded by the two other error components up to index $K_{\max }$, which is known a-priori in principle. A 'non-linearity dominance (blow-up)' may happened after that index. The optimal stopping index $N$ is roughly situated at the step when $e_{n}^{a p p}$ and $e_{n}^{n o i}$ are of the same order. priori by

The (modified) propagated data noise error in sIRGNM can be bounded a-

$$
\left\|e_{n}^{n o i}\right\| \leq \frac{\delta}{2 \sqrt{\alpha_{n}}}
$$


which is obtained by studying carefully the works of Mahale \& Nair [11], Jin [8] and [5]. This bound is also the same expression of the propagated noise error in IRGNM, where the rate of decay of the approximation error, $e_{n}^{a p p}$, depends on the smoothness of the unknown solution $a^{\sharp}$, to be precise on the smoothness of $\left(a^{\sharp}-a_{0}\right)$. With a slight modification, this is also true for sIRGNM, by utilizing the property of $F^{\prime}\left[a_{0}\right]$, which is likely known apriori. The essence of the Lepskij stopping rule is to extract information from the a-priori bound (2.1) to detect the point after which the propagated data error is become dominant.In the following theorem as given in [2], this situation stated precisely, as the proof is quite illustrative and short, we reproduce it here.

Theorem 2.1. [2] Let $a_{n}^{\delta}$ be the sequence of iterates produced by an iterative regularization method for an initial guess $a_{0}$ from some admissible set and data $\left(\delta, y^{\delta}\right)$ satisfying

We assume that

$$
u^{o b s}:=y^{\delta}=F\left(a^{\sharp}\right)+\delta \xi .
$$

- There exists an a-priori known index $K_{\max }=K_{\max }(\delta)$ such that $a_{n}^{\delta}$ is well defined for $0 \leq n \leq K_{\max }$.

- There exists an 'optimal' stopping index $N=N\left(\delta, y^{\delta}, a^{\sharp}\right) \in\left\{0,1, \cdots, K_{\max }\right\}$, and a known increasing function $\Phi: \mathrm{N}_{0} \rightarrow[0, \infty)$ such that

$$
\left\|a_{n}^{\delta}-a^{\sharp}\right\| \leq \Phi(n) \delta, \quad n=N, \cdots, K_{\max } .
$$

Then ther error at the Lepskij stopping index $n_{*}=n_{*}\left(\delta, y^{\delta}\right)$ defined by

$$
\begin{gathered}
n_{*}:=\min \left\{n \in\left\{0, \cdots, K_{\max }(\delta)\right\}:\left\|a_{n}^{\delta}-a^{\sharp}\right\| \leq 2 \Phi(m) \delta, \forall m=n+1, \cdots, K_{\max }\right\}, \\
\quad \text { is bounded by } \\
\left\|a_{n_{*}}^{\delta}-a^{\sharp}\right\| \leq 3 \Phi(N) \delta .
\end{gathered}
$$

Proof. Since $\Phi$ is increasing, we have

$$
\left\|a_{m}^{\delta}-a_{N}^{\delta}\right\| \leq\left\|a_{m}^{\delta}-a^{\sharp}\right\|+\left\|a_{N}^{\delta}-a^{\sharp}\right\| \leq \Phi(m) \delta+\Phi(N) \delta \leq 2 \Phi(m) \delta
$$

for $m=N+1, \cdots, K_{\max }(\delta)$. Consequently imply $n_{*} \leq N$. therefore,

$$
\left\|a_{n_{*}}^{\delta}-a^{\sharp}\right\| \leq\left\|a_{N}^{\delta}-a^{\sharp}\right\|+\left\|a_{N}^{\delta}-a_{n_{*}}^{\delta}\right\| \leq \Phi(N) \delta+2 \Phi(N) \delta \leq 3 \Phi(N) \delta,
$$

hence the assertion follows.

Some observations should be made in order to highlights the above theorem.

- The bound (2.1) and the remark following on the bound, lead us to choose $\Phi:=\kappa \alpha_{n}^{-1 / 2}$, for some constant $\kappa>1$.

- Within the algorithm, the optimal stopping index $N$ never appear explicitely. In the case of IRGNM, the explicit appearance of the optimal stopping index for prescribed data is not necessary, since it is determined by the some rule that a-priorily depends on the smoothness of $a^{\sharp}$. The smoothness of the solution with respect to the smoothing properties of non-linear operator $F$ usually expressed in terms of source conditions

$$
a_{0}-a^{\sharp}=\Lambda\left(F^{\prime}\left[a^{\sharp}\right]^{*} F^{\prime}\left[a^{\sharp}\right]\right) w, \quad\|w\| \leq \rho .
$$


Analogous to this result in IRGNM, in the case of sIRGNM, we replace the above source conditions, using modified source conditions

$$
a_{0}-a^{\sharp}=\Lambda\left(F^{\prime}\left[a_{0}\right]^{*} F^{\prime}\left[a_{0}\right]\right) w, \quad\|w\| \leq \rho .
$$

If $\Lambda(t)=t^{\mu}$, we refer to a Hoelder-type source condition, if $\Lambda(t)=$ $(\log (t))^{-p}$, we mean a logarithmic source condition.

- The maximum iteration is chosen $K_{\max }=C_{F}+s \log _{q} \frac{\delta}{\alpha_{0}}$, for some constant $C_{F} \in \mathrm{R}$, and $s=2$ if $F$ satisfies a stronger non-linearity condition.

\section{Inverse Source Problem.}

We consider the identification of the support of an external force acting over $\Omega \subset G$ from measurement of potential flux $\partial u / \partial n$ and the potential $u$ on the boundary $\Gamma=\partial G$. The situation can be rearranged such that $\left.u\right|_{\Gamma}=0$, by subtracting to a solution of the Laplace equation. Then the forward problem is described by the boundary value problem

$$
\Delta u=\chi_{\Omega}, \quad u=0 \text { on } \Gamma
$$

where the domain $\Omega$ is the support of $H$, and $\chi_{\Omega}=\operatorname{supp}(H)$ denoted the characteristic function of $\Omega$, which we assume to be star-shaped with respect to the origin. Then $\partial \Omega:=\{q(t)(\cos t, \sin t): t \in[0,2 \pi]\}$ for $q$ which is a positive function and $2 \pi$-periodic. The inverse problem consists in identifying the shape of $\Omega$ given the Neumann data $\frac{\partial u}{\partial n}$ of the solution on $\Gamma$. Therefore, we define $F$ as the operator mapping $q$ to $\frac{\partial u}{\partial n}$.

In this case we consider the identification of the shape of a unit constant external force on $\Omega \subset G$ from measurement of potential flux $\partial u / \partial n$ and the potential $u$ on the boundary $\Gamma=\partial G$.

It has been shown in [7] that logarithmic source conditions are equivalent to smoothness conditions in terms of Sobolev spaces if $\partial \Omega$ and $\Gamma$ are concentric circles.

Numerical Result. We assume that the data are $n$ noisy measurements of $g^{\sharp}=$ $F\left(q^{\sharp}\right)(t)$ at equidistant points $t_{j}^{(n)}:=\frac{j}{n}$,

$$
Y_{j}=g^{\sharp}\left(t_{j}^{(n)}\right)+\delta \xi_{j}, \quad j=1, \cdots, n,
$$

where $\delta$ is error level, and $\xi_{j}$ is randomly generated with $\|\xi\| \leq 1$.

For numerical test, we have chosen $\Gamma:=\{x:|x|=1\}$, and we used an exact solutions $a^{\sharp}:=q^{\sharp}(t):=0.5 *(1+0.9 \cos (t)+0.1 \sin (t)) /(1+0.75 \cos (t))$, a bean shaped inclusion.

The initial guess we choose $a^{0}:=q^{(0)}(t)=1$, a unit circular inclusion, and fixed $F^{\prime}\left[a^{0}\right]$.

We tested the rates of convergence with the balancing principle for the exact solution $a^{\sharp}$, by fixing $n=64$. 
The results in figure (3) show the Lepskij rule based on the worst case bound (2.1) and predicted optimal index (2.3). The performance of balancing principle tested first for $\kappa=1.1$ and $\kappa=0.3$.

FiguRE 1. Lepskij rule based on worst case bound. (Left column) Lepskij \& Optimal, L2-error versus Noise Level, and (Right column) reconstruction results with noise $5 \%$

Acknowledgement. This work funded by The Directorate General of Higher Education, Ministry of Education, The Government of Indonesia, under staff development programme in higher education establishment, years 2008-2010. The support by Graduirtenkolleg 1023 'Identification of Mathematical Models' at the Georg-August University of Goettingen, is here gratefully acknowledged.

\section{References}

[1] Bakushinsky,A.B. and Kokurin,M.Y. and Kokurin,M.I.U. and Smirnova,A., Iterative Methods for Ill-Posed Problems: An Introduction,(De Gruyter, Berlin, 2010)

[2] Bauer,F. And Hohage,T., A Lepskij-type stopping-Rule for regularized Newton-type methods, Inv. Problems, 21,1975, 2005.

[3] Bauer,F. And Hohage,T., A Lepskij-type Stopping-Rule for Newton-type Methods with Random Noise, PAMM, 5,15, 2005.

[4] Bauer,F. And Lukas,M.A., Comparing parameter choice methods for regularization of illposed problems, Mathematics and Computers in Simulation,81(9),1795, 2011.

[5] George,S., On convergence of regularized modified Newton's method for nonlinear ill-posed problems, J. Inv. and Ill-posed Prob.,18(2), 133, 2010.

[6] Bauer,F. and Hohage,T. And Munk,A. Iteratively Regularized Gauss-Newton Methods for Nonlinear Inverse Problem with Random Noise, SIAM J. Num.An., 47,1827,2009.

[7] Hohage, T., On the numerical solution of a three dimensional inverse medium scattering problem,Inv.Problem, 17, 1743, 2001.

[8] JiN, Q.N. On a class of frozen regularized Gauss-Newton methods for nonlinear inverse problems, Math. Comp., 79, 2191, 2010.

[9] Kaltenbacher,B., A posteriori parameter choice strategies for some Newton type methods for the regularization of nonlinear ill-posed problems, Num. Math., v 79, 501, 1998.

[10] Kaltenbacher,B. and Neubauer,A. and Scherzer,O., Iterative Regularization Methods for Nonlinear Ill-Posed Problems, (de Gruyter, Berlin, 2008).

[11] Mahale, P. And NaIR,M.T., A simplified generalized Gauss-Newton method for nonlinear ill-posed problems, Math. Comp., 78, 171-184, 2009.

Agah D. Garnadi: Dept. of Mathematics

Institut Pertanian Bogor.

E-mails: agah.garnadi@gmail.com 